\title{
Amplify-and-Forward Relay Transmission with End-to-End Antenna Selection
}

\author{
Himal A. Suraweera*, George K. Karagiannidis $\dagger$, Yonghui Li $\ddagger$, Hari K. Garg*, A. Nallanathan $\S$ and Branka Vucetic $\ddagger$ \\ * Department of Electrical and Computer Engineering, National University of Singapore, Singapore

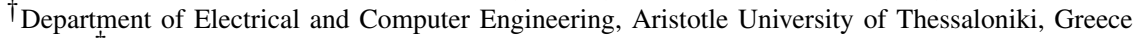

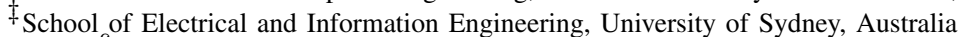

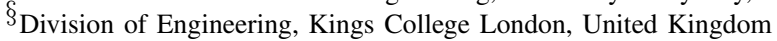 \\ E-mail: \{elesaha, eleghk\}@nus.edu.sg, geokarag@auth.gr, \{lyh,branka\}@ee.usyd.edu.au, arumugam.nallanathan@kcl.ac.uk
}

\begin{abstract}
In this paper, the performance of a dual-hop Amplify-and-Forward (AF) multi-antenna relay network, with end-to-end (e2e) best antenna selection, is investigated. To investigate the performance of this system, we first derive the exact outage probability in closed-form. It is then used to obtain expressions for e2e SNR moments and the average symbol/bit error rate (SER/BER) valid for a large class of practical modulation schemes. A simple and accurate BER approximation is also derived to quantify the performance at high SNR. Our analytical results, show that the e2e antenna pair selection scheme achieves the same diversity order as for the case where all antennas are used. To further confirm the validity of our analysis, Monte Carlo simulation results are also presented.
\end{abstract}

\section{INTRODUCTION}

Future wireless networks will support relay based communications since they offer significant performance benefits, including increased spatial diversity, capacity and extended coverage [1], [2]. One of the most commonly used relaying protocols is Amplify-and-Forward (AF). Some AF relays use the source to relay channel knowledge for amplifying the received signal. These kind of relays are in general known as channel state information (CSI)-assisted relays.

In the available literature, various performance aspects of dual-hop relay networks have been investigated. While most of such performance studies assume single antennas at each node, several recently published papers consider multiple antenna deployments at the source/relay and the destination (see, for e.g. [3]-[10]). In [3], the impact of receive diversity on the symbol error rate (SER) performance of a fixed gain and CSI-assisted relay network has been investigated. In [4], the network capacity of several signalling and routing methods for multiple-input multiple-output (MIMO) relay systems has been compared. In [5], the impact of multiple antennas on the performance of a decode-and-forward (DF) distributed cooperative network has been examined. In [6], Louie et al. have analyzed a dual-hop CSI-assisted AF relay network with beamforming at the source and maximal ratio combining (MRC) at the destination providing exact and asymptotic error performance results. Assuming fixed-gain AF relays, the performance of the same network has been studied by Costa and Aissa in [7]. More recently, antenna selection techniques in

This research work was supported by the National University of Singapore under Research Grant Numbers R-263-000-421-112 \& R-263-000-436-112. multi-antenna relay systems has also come under consideration [8]-[10]. In [8], optimal SNR-based transmit antenna selection rules at the source/relay nodes for the AF half-duplex MIMO relay channel, have been derived. In [9], the performance of a dual-hop AF relay system with transmit antenna selection at the source and MRC at the destination has been investigated.

In this paper, a dual-hop AF MIMO relay network with the best antenna selected at each end is considered. This system has a reduced performance compared to the transmit beamforming/MRC system studied in [6]. However, this simple antenna selection method requires much less feedback overhead [8]. In [10], assuming an ideal relay gain, the probability density function (pdf) of the system's e2e SNR has been derived. However, in [10] other important performance metrics of the system has not been presented. This paper fills this gap. We have derived closed-form expressions for the exact outage probability, moments of the e2e SNR and the error performance including the average bit error rate (BER) for various modulations and the average SER of $M$-QAM. To gain further valuable insights into the system performance at high SNR, we also present asymptotic SER/BER approximations which have also not been investigated in [10].

The rest of the paper is organized as follows. In Section II, the dual-hop AF system model with best antenna selection is described. Closed-form expressions for the statistics of the e2e SNR are presented in Section III and in Section IV, the average error performance, diversity and array gains are investigated. We then proceed by verifying the new results in Section V. Finally, conclusions are drawn in Section VI.

\section{System AND Channel Model}

We consider a wireless communications system where a source, $S$, equipped with $N_{S}$ antennas communicates with the destination, $D$, equipped with $N_{D}$ antennas, through a relay, $R$, equipped with $N_{R}$ antennas. It is assumed that $S$ does not have a direct link to $D$. Hence, in this network, the diversity gain expected from the use of multiple antennas is of significant interest since cooperative diversity can no longer be achieved.

The system operation is as follows: the source and the relay transmit on orthogonal channels. We consider a time multiplexing scheme, for which communications from $S$ to $D$ takes 
places in two time slots. An $N_{S} \times N_{R}$ matrix, $\mathbf{H}_{S R}$, is used to denote the channel paths between the source and the relay and an $N_{D} \times N_{R}$ matrix, $\mathbf{H}_{R D}$, is used to denote the channel paths between the relay and the destination. Their entries are modeled as i.i.d complex Gaussian random variables (RVs) corresponding to Rayleigh fading. Prior to sending data, with the help of pilot symbols, at each hop, a single transmit/receive antenna pair that maximizes the received SNR is selected. The same relay system model has been considered in [10] and is an extension of the point-to-point system first studied in [11]. We assume that the destination has perfect channel knowledge of the selected $S-R$ and $R-D$ antenna paths for coherent decoding of the signals.

The received signal at the relay during the first time slot can be written as

$$
y_{R}=\sqrt{P_{1}} h_{1} x+n_{R},
$$

where $h_{1}$ is the complex channel between the selected source transmit antenna to the relay receive antenna, $P_{1}$ is the transmit power, $x$ is the transmitted scalar symbol with zero mean and unit variance, $n_{R}$ is the additive white Gaussian noise (AWGN) satisfying $E\left[\left|n_{R}\right|^{2}\right]=\sigma_{1}^{2}$. The received scalar signal at the relay is then multiplied by a gain $G$, and transmitted to the destination during the second time slot. The received signal at the destination can be written as

$$
y_{D}=\sqrt{P}_{2} h_{2} G\left(\sqrt{P}_{1} h_{1} x+n_{R}\right)+n_{D},
$$

where $P_{2}$ is the relay transmit power, $h_{2}$ is the complex Rayleigh channel between the selected relay transmit antenna to the destination receive antenna and $n_{D}$ is the AWGN satisfying $E\left[\left|n_{D}\right|^{2}\right]=\sigma_{2}^{2}$. According to the principles of CSIassisted AF relaying, the gain $G$ is chosen as [2]

$$
G^{2}=\frac{1}{P_{1}\left|h_{1}\right|^{2}+\sigma_{1}^{2}}
$$

and the e2e SNR is of the form:

$$
\gamma_{e q 1}=\frac{\gamma_{1} \gamma_{2}}{\gamma_{1}+\gamma_{2}+1}
$$

where $\gamma_{1}=\left|h_{1}\right|^{2} \bar{\gamma}_{1}, \gamma_{2}=\left|h_{2}\right|^{2} \bar{\gamma}_{2}$, and $\bar{\gamma}_{i}=\frac{P_{i}}{\sigma_{i}^{2}}$ for $i=$ 1,2 . However, the e2e SNR in (4) is not easily tractable [2]. Fortunately, (4) can be tightly upper bounded by

$$
\gamma_{e q 1}<\gamma_{e q 2}=\frac{\gamma_{1} \gamma_{2}}{\gamma_{1}+\gamma_{2}} .
$$

The form of $\gamma_{e q 2}$ in (5) has the advantage of mathematical tractability over that in (4) and it is a tight upper bound for $\gamma_{e q 1}$, specially at medium to high average SNR.

\section{Outage Probability and SNR Moments}

In this section, we derive important performance measures for the dual-hop relay system under investigation. This includes the outage probability, asymptotic outage probability and the e2e SNR moments.
To study the outage probability of (4) and (5), it is necessary to obtain the distribution of the e2e SNR, of the RV

$$
Z=\frac{\gamma_{1} \gamma_{2}}{\gamma_{1}+\gamma_{2}+c}
$$

where $c \geq 0$ is a constant.

\section{A. Outage Probability}

The outage probability, $P_{o}$, defined as the probability that $\gamma_{e q 1}$ drops below an acceptable SNR threshold $\gamma_{T}$, is an important quality of service (QoS) measure. Mathematically,

$$
P_{o}=\operatorname{Pr}\left(\gamma_{e q 1}<\gamma_{T}\right)=F_{\gamma_{e q 1}}\left(\gamma_{T}\right) .
$$

To derive the outage probability of $\gamma_{e q 1}$, we first present a theorem for the cumulative distribution function (cdf) and the pdf of the RV $Z$, in (6).

Theorem 1: The cdf and pdf of $Z$ are given respectively by

$$
\begin{aligned}
F_{Z}(z) & =1-2 N_{t} N_{r} \sqrt{\frac{z^{2}+c z}{\bar{\gamma}_{1} \bar{\gamma}_{2}}} \sum_{l=0}^{N_{t}-1} \frac{(-1)^{l}\left(\begin{array}{c}
N_{t}-1 \\
l
\end{array}\right)}{\sqrt{1+l}} \\
& \times e^{-\frac{(1+l) z}{\bar{\gamma}_{1}}} \sum_{k=0}^{N_{r}-1} \frac{(-1)^{k}\left(\begin{array}{c}
N_{r}-1 \\
k
\end{array}\right)}{\sqrt{1+k}} e^{-\frac{(1+k) z}{\gamma_{2}}} \\
& \times K_{1}\left(2 \sqrt{\frac{(1+l)(1+k)\left(z^{2}+c z\right)}{\bar{\gamma}_{1} \bar{\gamma}_{2}}}\right)
\end{aligned}
$$

and

$$
\begin{aligned}
& p_{Z}(z)=2 N_{t} N_{r} \sqrt{\frac{z^{2}+c z}{\bar{\gamma}_{1} \bar{\gamma}_{2}}} \sum_{l=0}^{N_{t}-1} \frac{(-1)^{l}\left(\begin{array}{c}
N_{t}-1 \\
l
\end{array}\right)}{\sqrt{1+l}} \\
& \times e^{-\frac{(1+l) z}{\bar{\gamma}_{1}}} \sum_{k=0}^{N_{r}-1} \frac{(-1)^{k}\left(\begin{array}{c}
N_{r}-1 \\
k
\end{array}\right)}{\sqrt{1+k}} e^{-\frac{(1+k) z}{\bar{\gamma}_{2}}}((2 z+c) \\
& \times \sqrt{\frac{(1+l)(1+k)}{\left(z^{2}+c z\right) \bar{\gamma}_{1} \bar{\gamma}_{2}}} K_{0}\left(2 \sqrt{\frac{(1+l)(1+k)\left(z^{2}+c z\right)}{\bar{\gamma}_{1} \bar{\gamma}_{2}}}\right) \\
& \left.+\left(\frac{1+l}{\bar{\gamma}_{1}}+\frac{1+k}{\bar{\gamma}_{2}}\right) K_{1}\left(2 \sqrt{\frac{(1+l)(1+k)\left(z^{2}+c z\right)}{\bar{\gamma}_{1} \bar{\gamma}_{2}}}\right)\right),
\end{aligned}
$$

where $N_{t}=N_{S} N_{R}, N_{r}=N_{R} N_{D}$ and $K_{\nu}(x)$ is the $\nu$-th order modified Bessel function of the second kind [12, Eq. (8.432.6)].

Proof: The proof is given in the Appendix.

We now present the following corollary for the outage probability and the e2e SNR pdf of the system.

Corollary 1: The outage probability and the e2e SNR pdf of the system can now be obtained by first substituting $z=\gamma_{T}$ and $z=\gamma$ into (8) and (9) respectively. The outage probability and SNR pdf of the system follow by substituting $c=1$ into the resultant expressions.

\section{B. Outage Probability at High SNR}

We now analyze the system's e2e asymptotic outage probability. 
Theorem 2: The outage probability in the large SNR regime is given by

$$
P_{o}^{\infty} \simeq \begin{cases}\left(\frac{\gamma_{T}}{\bar{\gamma}_{1}}\right)^{N_{t}}+O\left(\frac{\gamma_{T}}{\bar{\gamma}_{1}}\right)^{N_{t}} & N_{t}<N_{r} \\ \left(1+\frac{1}{\mu^{N}}\right)\left(\frac{\gamma_{T}}{\bar{\gamma}_{1}}\right)^{N}+O\left(\frac{\gamma_{T}}{\bar{\gamma}_{1}}\right)^{N} & N_{t}=N_{r}=N \\ \frac{1}{\mu^{N_{r}}}\left(\frac{\gamma_{T}}{\bar{\gamma}_{1}}\right)^{N_{r}}+O\left(\frac{\gamma_{T}}{\bar{\gamma}_{1}}\right)^{N_{r}} & N_{t}>N_{r}\end{cases}
$$

where $\mu=\frac{\bar{\gamma}_{2}}{\bar{\gamma}_{1}}$ and a function of $x, f(x)$, is represented as $O(x)$ if $\lim _{x \rightarrow 0} \frac{f(x)}{x}=0$.

Proof: The proof is given in the Appendix.

Note, that the presented expression in (10) is much simpler compared to (8) and allows us to study important factors such as the diversity gain of the system.

\section{Moments of the e2e SNR}

We now characterize the moments of $\gamma_{e q 2}$ given in (5). The moments of the e2e SNR are important performance metrics directly related to the system's performance [6], [7]. It can be showed that the moments of $\gamma_{e q 2}$ are given by

$$
\mathbb{E}\left[\gamma_{\text {eq2 }}^{n}\right]=n \int_{0}^{\infty} \gamma^{n-1}\left(1-F_{\gamma_{\text {eq2 }}}(\gamma)\right) d \gamma,
$$

where $F_{\gamma_{e q 2}}(\cdot)$ denote the cdf of $\gamma_{e q 2}$ and $\mathbb{E}(\cdot)$ is the expectation operator. After substituting (8) with $c=0$ into (11) and using [12, Eq. (6.621.3)], the moments of the e2e can be evaluated in closed-form. Therefore, $E\left[\gamma_{e q 2}^{n}\right]$ is expressed as

$$
\begin{aligned}
& \mathbb{E}\left[\gamma_{e q 2}^{n}\right]=\frac{8 \sqrt{\pi} N_{t} N_{r} n !(n+1) !}{\bar{\gamma}_{1} \bar{\gamma}_{2}} \sum_{l=0}^{N_{t}-1}(-1)^{l}\left(\begin{array}{c}
N_{t}-1 \\
l
\end{array}\right) \\
& \times \sum_{k=0}^{N_{r}-1}(-1)^{k}\left(\begin{array}{c}
N_{r}-1 \\
k
\end{array}\right) \\
& \times \frac{{ }_{2} F_{1}\left(n+2, \frac{3}{2} ; n+\frac{3}{2} ; \frac{\frac{1+l}{\bar{\gamma}_{1}}+\frac{1+k}{\bar{\gamma}_{2}}-2 \sqrt{\frac{(1+l)(1+k)}{\bar{\gamma}_{1} \bar{\gamma}_{2}}}}{\Gamma\left(n+\frac{3}{2}\right)\left(\frac{1+l}{\bar{\gamma}_{1}+\frac{1+k}{\bar{\gamma}_{2}}+2 \sqrt{\frac{\left(1+l_{1}(1+k)\right.}{\bar{\gamma}_{1} \bar{\gamma}_{2}}}}\right)}\right)}{\left.\overline{\bar{\gamma}}_{2}+2 \sqrt{\frac{(1+l)(1+k)}{\bar{\gamma}_{1} \bar{\gamma}_{2}}}\right)^{n+2}},
\end{aligned}
$$

where $\Gamma(x)$ is the gamma function and ${ }_{2} F_{1}(a, b ; c ; z)$ is the Gauss hypergeometric function [12].

\section{Average Error Performance}

In this section, we derive closed-form expressions for the e2e average BER of the system under consideration for various modulation formats. Although in [10], an average BER expression for $M$-PSK modulation has been derived, it is in the form of an integral expression. We consider $\gamma_{e q 2}$ instead of (4) for mathematical tractability purposes and because it provides a tight upper bound for CSI-assisted AF relaying in the medium to high SNR regime. Our results apply for all modulation formats that have a BER expression of the form:

$$
P_{b}=a \mathbb{E}_{\gamma}[Q(\sqrt{b \gamma})],
$$

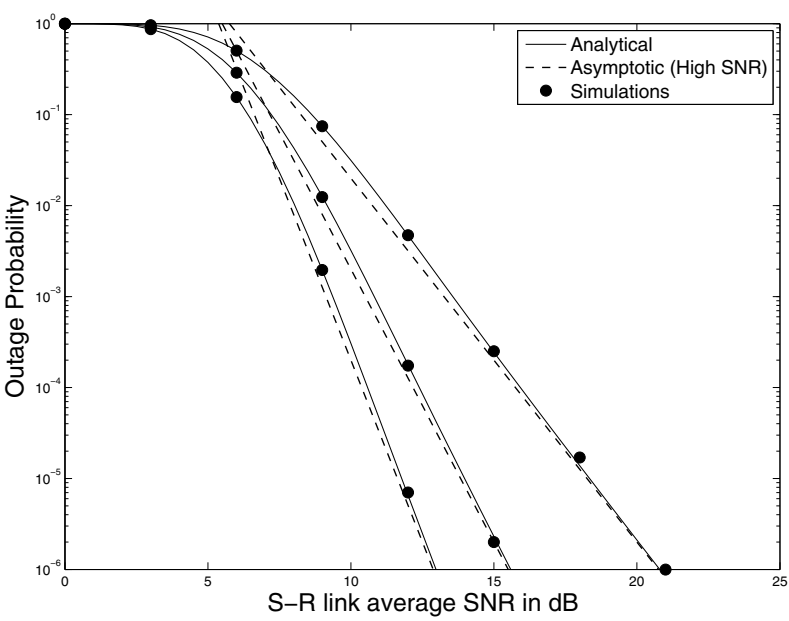

Fig. 1. Outage probability against $\bar{\gamma}_{1}$ for various antenna configurations. $N_{S}=N_{D}=2,3,4$ and $N_{R}=2 . \gamma_{T}=5 \mathrm{~dB}$ and $\bar{\gamma}_{2}=\bar{\gamma}_{1}$.

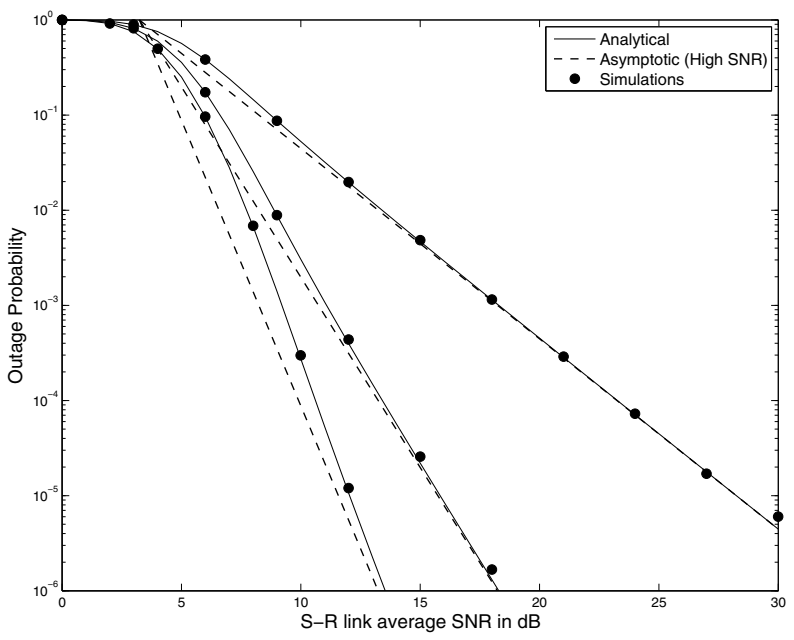

Fig. 2. Outage probability against $\bar{\gamma}_{1}$ for various antenna configurations. $N_{S}=4, N_{R}=2$ and $N_{D}=1,2,3 . \gamma_{T}=5 \mathrm{~dB}$ and $\bar{\gamma}_{2}=1.5 \bar{\gamma}_{1}$.

where $a, b>0$ and $Q(x)=\frac{1}{\sqrt{2 \pi}} \int_{x}^{\infty} e^{-\frac{y^{2}}{2}} d y$ is the Gaussian $Q$-function.

Consider the following integral defined as

$$
T(a, b, s)=\frac{a}{\sqrt{2 \pi}} \int_{0}^{\infty} F_{\gamma_{e q 2}}\left(\frac{t^{2}}{b}\right) e^{-s t^{2}} d t .
$$

Substituting (8) into (14), $T(a, b, s)$ can be expressed as

$$
\begin{aligned}
& T(a, b, s)=\frac{a}{\sqrt{2 \pi}} \int_{0}^{\infty} e^{-s t^{2}} d t-\frac{a N_{t} N_{r}}{b} \sqrt{\frac{2}{\pi \bar{\gamma}_{1} \bar{\gamma}_{2}}} \\
& \times \sum_{l=0}^{N_{t}-1} \frac{(-1)^{l}\left(\begin{array}{c}
N_{t}-1 \\
l
\end{array}\right)}{\sqrt{1+l}} \sum_{k=0}^{N_{r}-1} \frac{(-1)^{k}\left(\begin{array}{c}
N_{r}-1 \\
k
\end{array}\right)}{\sqrt{1+k}} \\
& \times \int_{0}^{\infty} t^{2} e^{-\left(\frac{1+l}{b \bar{\gamma}_{1}}+\frac{1+k}{b \bar{\gamma}_{2}}+s\right) t^{2}} K_{1}\left(\frac{2 t^{2}}{b} \sqrt{\frac{(1+l)(1+k)}{\bar{\gamma}_{1} \bar{\gamma}_{2}}}\right) d t
\end{aligned}
$$

With the help of $\int_{0}^{\infty} e^{-q x^{2}} d x=\frac{1}{2} \sqrt{\frac{\pi}{q}}$, and employing [12, Eq. (6.621.3)], $T(a, b, s)$ has a closed-form solution given by (16) at the top of the next page. 


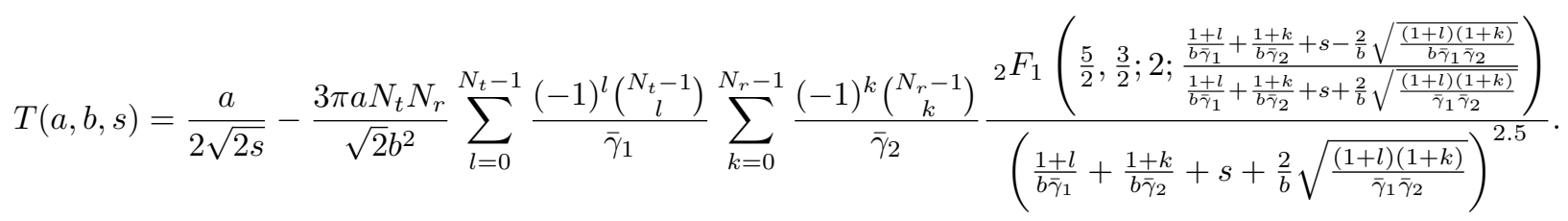

Now, using integration by parts, it can be shown that the average BER in (13) can be rewritten as

$$
P_{b}=T\left(a, b, \frac{1}{2}\right) \text {. }
$$

\section{A. Average SER of $M-Q A M$}

The classical form of the average SER for $M$-ary quadrature amplitude modulation (M-QAM) is given by

$$
P_{s}=a \mathbb{E}_{\gamma}[Q(\sqrt{b \gamma})]-a\left(1-\frac{1}{\sqrt{M}}\right) \mathbb{E}_{\gamma}\left[Q^{2}(\sqrt{b \gamma})\right]
$$

where $a=4(1-1 / \sqrt{M})$ and $b=3 /(M-1)$. Therefore, in order to derive the average SER we need to evaluate

$$
I(a, b)=a \mathbb{E}_{\gamma}\left[Q^{2}(\sqrt{b \gamma})\right] .
$$

After integration by parts (19) can be rewritten as

$$
I(a, b)=\frac{\sqrt{2} a}{\sqrt{\pi}} \int_{0}^{\infty} Q(u) F_{\gamma_{e q 2}}\left(\frac{u^{2}}{b}\right) e^{-\frac{u^{2}}{2}} d u .
$$

We are unaware of a closed-form solution to this integral. Nevertheless, using a $Q$-function tight approximation given by [15, Eq. (14)]

$$
Q(x) \simeq \frac{1}{12} e^{-\frac{x^{2}}{2}}+\frac{1}{4} e^{-\frac{2 x^{2}}{3}},
$$

$I(a, b)$ can be efficiently evaluated. Substituting (21) into (20) we get

$$
\begin{aligned}
I(a, b) & =\frac{a}{6 \sqrt{2 \pi}} \int_{0}^{\infty} F_{\gamma_{e q 2}}\left(\frac{u^{2}}{b}\right) e^{-u^{2}} d u \\
& +\frac{a}{2 \sqrt{2 \pi}} \int_{0}^{\infty} F_{\gamma_{e q 2}}\left(\frac{u^{2}}{b}\right) e^{-\frac{7}{6} u^{2}} d u .
\end{aligned}
$$

Therefore, $P_{s}$ can be written as

$$
\begin{aligned}
P_{s} & \simeq T\left(a, b, \frac{1}{2}\right)-\frac{1}{2}\left(1-\frac{1}{\sqrt{M}}\right) \\
& \times\left(\frac{T(a, b, 1)}{3}+T\left(a, b, \frac{7}{6}\right)\right) .
\end{aligned}
$$

\section{B. Diversity and Array Gain at High SNR}

At sufficiently high SNRs, diversity gain defines the slope of the average BER against average SNR in a log-log scale while $G_{a}$ in dB determines the shift of the curve with respect to the average BER curve of $\bar{\gamma}_{1}^{-G_{d}}$. The average BER at high SNRs can be closely approximated as [13, Prop. 1]

$$
P_{b}^{\infty}=\frac{2^{q} a \xi \Gamma\left(q+\frac{3}{2}\right)}{\sqrt{\pi}}\left(b \bar{\gamma}_{1}\right)^{-(q+1)}+O\left(\bar{\gamma}_{1}^{-(q+1)}\right),
$$

where $q=\min \left(N_{t}, N_{r}\right)-1$ and

$$
\xi= \begin{cases}N_{t} & N_{t}<N_{r} \\ \left(1+\frac{1}{\mu^{N}}\right) N & N_{t}=N_{r}=N \\ \frac{N_{r}}{\mu^{N_{r}}} & N_{t}>N_{r}\end{cases}
$$

Eq. (24) implies that the array gain $G_{a}$ and the diversity gain $G_{d}$ of the system can be written as

$$
G_{a}=b\left(\frac{2^{q} a \xi \Gamma\left(q+\frac{3}{2}\right)}{\sqrt{\pi}}\right)^{-\frac{1}{q+1}},
$$

and

$$
G_{d}=\min \left(N_{t}, N_{r}\right) .
$$

From (27) we can infer that selecting the best antenna pair at each end can achieve the maximum possible diversity order of this system.

We now present an approximation for the average SER of $M$-QAM modulation at high SNR. Using (10) and after some manipulations $I(a, b)$ at high SNR can be expressed as

$$
\begin{aligned}
I^{\infty}(a, b) & =\frac{2^{q} a \xi}{\sqrt{\pi}(q+1)} \int_{0}^{\infty} u^{2(q+1)} \operatorname{erfc}(u) e^{-u} d u \\
& \times\left(b \bar{\gamma}_{1}\right)^{-(q+1)}+O\left(\bar{\gamma}_{1}^{-(q+1)}\right),
\end{aligned}
$$

where $\operatorname{erfc}(\cdot)$ is the complementary error function. With the aid of [14, Eq. (2.8.5.7)] the integral in (28) can be evaluated in closed-form as follows

$$
\begin{aligned}
I^{\infty}(a, b) & =\frac{2^{q} q ! a \xi}{\pi(2 q+3)}{ }_{2} F_{1}\left(q+\frac{3}{2}, q+2 ; q+\frac{5}{2} ;-1\right) \\
& \times\left(b \bar{\gamma}_{1}\right)^{-(q+1)}+O\left(\bar{\gamma}_{1}^{-(q+1)}\right) .
\end{aligned}
$$

Finally, using (24) and (29) the average SER for $M$-QAM in the high SNR regime can be expressed as

$$
\begin{aligned}
P_{S}^{\infty} & =\frac{2^{q} a \xi}{\sqrt{\pi}}\left(\frac{\Gamma\left(q+\frac{3}{2}\right)}{q+1}-\left(1-\frac{1}{\sqrt{M}}\right) \frac{q !}{\sqrt{\pi}(2 q+3)}\right. \\
& \left.\times{ }_{2} F_{1}\left(q+\frac{3}{2}, q+2 ; q+\frac{5}{2} ;-1\right)\right)\left(b \bar{\gamma}_{1}\right)^{-(q+1)} .
\end{aligned}
$$

\section{Numerical and Simulation Results}

We have confirmed the correctness of the derived analytical results in Section III and IV, through comparison with Monte Carlo simulations. Figs. 1 and 2 show the e2e SNR outage for various numbers of antennas at $S$ and $D$. We see that the outage probability is significantly improved as the number of deployed antennas increase. In order to verify our analysis at high SNRs, we have also plotted the outage curves obtained 


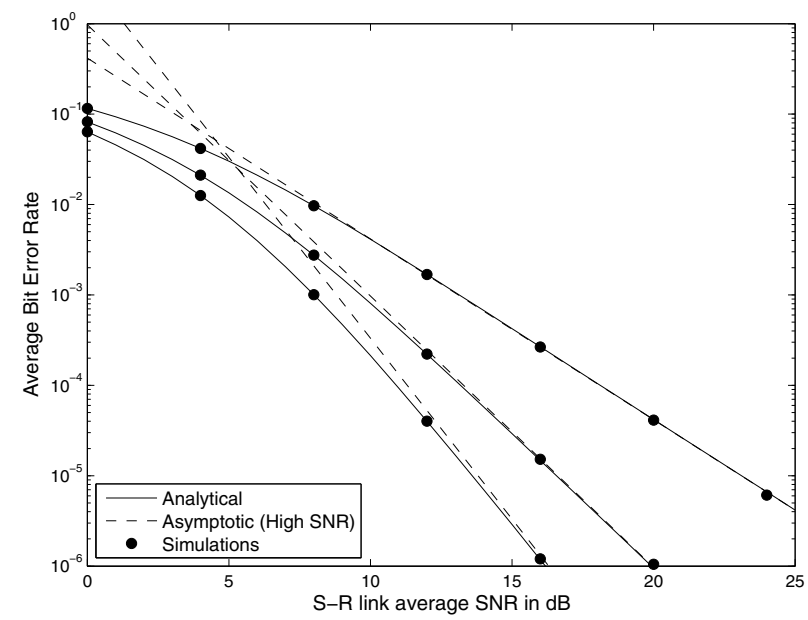

Fig. 3. Average BER with BPSK and $\bar{\gamma}_{2}=3 \bar{\gamma}_{1} . N_{S}=N_{D}=2,3,4$ and the relay is equipped with a single antenna.

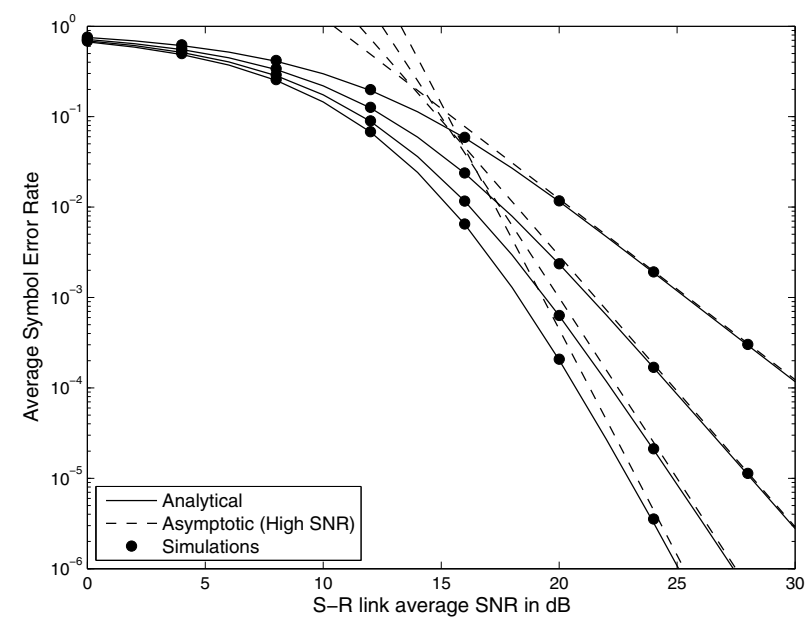

Fig. 4. Average SER with 16-QAM and $\bar{\gamma}_{2}=3 \bar{\gamma}_{1} . N_{S}=N_{D}=2,3,4,5$ and the relay is equipped with a single antenna.

from (10). It is apparent that these results are able to accurately predict system's outage at medium to high SNR region.

Due to space limitations, results of the system's e2e SNR moments are not presented. However, extensive simulations again confirmed the correctness of (12). In Figs. 3 and 4 we have evaluated the average BER and average SER versus SNR performance for BPSK and 16-QAM modulations, respectively. These figures clearly demonstrate the accuracy of the error performance analysis using (17) and (23), in comparison with the results obtained from simulations. We have also verified the accuracy of the high SNR approximations. In the case of BPSK, the exact and the asymptotic average BER results shown in Fig. 3 are almost indistinguishable when $\bar{\gamma}_{1}>15 \mathrm{~dB}$. In all cases, increasing the number of antennas has a positive impact on the error performance. However, as seen from Fig. 4 increasing the number of antennas higher than five, may not produce further significant performance gains. We note that increasing the number of deployed antennas also contribute to high infrastructure costs.

\section{Conclusions}

Antenna selection methods provide a good cost/performance tradeoff. The performance of a dual-hop AF relay system with e2e best antenna selection over Rayleigh fading channels was investigated. A range of closed-form results has been derived to evaluate the outage probability, the e2e SNR moments and the average BER/SER of the system. In order to gain further insights, asymptotic results were also derived including the array and diversity gains at high SNRs. The asymptotic analysis confirms that the system achieves the maximum possible diversity order, which is equal to the minimum of the product of the number of antennas available at the source/relay and relay/destination, respectively.

\section{APPENDIX}

\section{A. Proof of Theorem I}

The cdf of $Z$ can be written as

$$
F_{Z}(z)=\int_{0}^{\infty} \operatorname{Pr}\left(\frac{x \gamma_{1}}{\gamma_{1}+x+c}<z\right) p_{\gamma_{2}}(x) d x
$$

After applying some algebraic manipulations to (31), we obtain

$$
F_{Z}(z)=1-\int_{0}^{\infty} P_{\gamma_{1}}\left(z+\frac{z^{2}+c z}{w}\right) p_{\gamma_{2}}(z+w) d w
$$

where $P_{\gamma_{1}}(x)=1-F_{\gamma_{1}}(x)$ denotes the complementary cdf of $\gamma_{1}$ and $p_{\gamma_{2}}(x)$ is the pdf of $\gamma_{2}$.

To obtain the cdf of $Z$, we need the cdf of $\gamma_{1}$ and the pdf of $\gamma_{2}$. Note that the cdf of $\gamma_{1}$ is given by

$$
F_{\gamma_{1}}(x)=1-N_{t} \sum_{l=0}^{N_{t}-1} \frac{(-1)^{l}}{1+l}\left(\begin{array}{c}
N_{t}-1 \\
l
\end{array}\right) e^{-\frac{(1+l) x}{\gamma_{1}}},
$$

and the pdf of $\gamma_{2}$ is given by

$$
p_{\gamma_{2}}(x)=\frac{N_{r}}{\bar{\gamma}_{2}} \sum_{k=0}^{N_{r}-1}(-1)^{k}\left(\begin{array}{c}
N_{r}-1 \\
k
\end{array}\right) e^{-\frac{(1+k) x}{\bar{\gamma}_{2}}}
$$

Substituting (33) and (34) into (32), solving the resultant integral using [12], and after some algebraic manipulations, we arrive at the desired result. By taking the derivative of (8) with respect to $z$, taking into account that $\frac{d K_{1}(x)}{d x}=$ $-K_{0}(x)-\frac{K_{1}(x)}{x}$, we obtain the pdf of $Z$.

\section{B. Proof of Theorem II}

To obtain an expansion of $F_{\gamma_{e q 1}}\left(\gamma_{T}\right)$, we first note that

$$
\lim _{\bar{\gamma}_{1}, \bar{\gamma}_{2} \rightarrow \infty} F_{\gamma_{e q 1}}\left(\gamma_{T}\right)=\lim _{\bar{\gamma}_{1}, \bar{\gamma}_{2} \rightarrow \infty} F_{\gamma_{e q 2}}\left(\gamma_{T}\right)
$$

We substitute $\bar{\gamma}_{2}=\mu \bar{\gamma}_{1}$ and $c=0$ into (8) and write the outage probability as

$$
\begin{aligned}
F_{\gamma_{e q 2}}\left(\gamma_{T}\right) & =1-\frac{2 \gamma_{T}}{\sqrt{\mu} \bar{\gamma}_{1}} N_{t} N_{r} \sum_{l=0}^{N_{t}-1} \frac{(-1)^{l}\left(\begin{array}{c}
N_{t}-1 \\
l
\end{array}\right)}{\sqrt{1+l}} \\
& \times e^{-\frac{(1+l) \gamma_{T}}{\bar{\gamma}_{1}}} \sum_{k=0}^{N_{r}-1} \frac{(-1)^{k}\left(\begin{array}{c}
N_{r}-1 \\
k
\end{array}\right)}{\sqrt{1+k}} e^{-\frac{(1+k) \gamma_{T}}{\mu \bar{\gamma}_{1}}} \\
& \times K_{1}\left(\frac{2 \gamma_{T}}{\bar{\gamma}_{1}} \sqrt{\frac{(1+l)(1+k)}{\mu}}\right)
\end{aligned}
$$


Now by substituting $x=\frac{\gamma_{T}}{\bar{\gamma}_{1}}$ we can rewrite (36) as

$$
\begin{aligned}
F_{\gamma_{e q 2}}(x) & =1-\frac{2 N_{t} N_{r}}{\sqrt{\mu}} x \sum_{l=0}^{N_{t}-1} \frac{(-1)^{l}\left(\begin{array}{c}
N_{t}-1 \\
l
\end{array}\right)}{\sqrt{1+l}} \\
& \times e^{-(1+l) x} \sum_{k=0}^{N_{r}-1} \frac{(-1)^{k}\left(\begin{array}{c}
N_{r}-1 \\
k
\end{array}\right)}{\sqrt{1+k}} e^{-\frac{(1+k) x}{\mu}} \\
& \times K_{1}\left(2 x \sqrt{\frac{(1+l)(1+k)}{\mu}}\right) .
\end{aligned}
$$

Next, employing an approximation for small arguments of $x$, $0<x<<\sqrt{\alpha+1}$, given by

$$
K_{\alpha}(x) \simeq \frac{2^{\alpha-1} \Gamma(\alpha)}{x^{\alpha}}
$$

$F_{\gamma_{e q 2}}(x)$ can be simplified as

$$
\begin{aligned}
F_{\gamma_{e q 2}}(x) & \simeq 1-N_{t} N_{r} \sum_{l=0}^{N_{t}-1} \frac{(-1)^{l}\left(\begin{array}{c}
N_{t}-1 \\
l
\end{array}\right)}{1+l} \\
& \times \sum_{k=0}^{N_{r}-1} \frac{(-1)^{k}\left(\begin{array}{c}
N_{r}-1 \\
k
\end{array}\right)}{1+k} e^{-\left(1+l+\frac{1+k}{\mu}\right) x} .
\end{aligned}
$$

Using the McLaurin series representation for the exponential function in (39) yields

$$
\begin{aligned}
F_{\gamma_{e q 2}}(x) & \simeq-N_{t} N_{r} \sum_{l=0}^{N_{t}-1} \frac{(-1)^{l}\left(\begin{array}{c}
N_{t}-1 \\
l
\end{array}\right)}{1+l} \\
& \times \sum_{k=0}^{N_{r}-1} \frac{(-1)^{k}\left(\begin{array}{c}
N_{r}-1 \\
k
\end{array}\right)}{1+k} \sum_{\kappa=1}^{\infty} \frac{\left(-\left(1+l+\frac{1+k}{\mu}\right) x\right)^{\kappa}}{\kappa !} .
\end{aligned}
$$

Now, applying the Binomial expansion to $\left(1+l+\frac{1+k}{\mu}\right)^{\kappa}$ and realizing that the first or the second summation vanishes for $\kappa<\min \left(N_{t}, N_{r}\right)$, (40) simplifies to

$$
\begin{aligned}
& F_{\gamma_{e q 2}}(x) \simeq-N_{t} N_{r}(-x)^{\min \left(N_{t}, N_{r}\right)} \sum_{l=0}^{N_{t}-1} \frac{(-1)^{l}\left(\begin{array}{c}
N_{t}-1 \\
l
\end{array}\right)}{1+l}(41) \\
& \times \sum_{k=0}^{N_{r}-1} \frac{(-1)^{k}\left(\begin{array}{c}
N_{r}-1 \\
k
\end{array}\right)}{1+k} \frac{(1+l)^{\min \left(N_{t}, N_{r}\right)}+\left(\frac{1+k}{\mu}\right)^{\min \left(N_{t}, N_{r}\right)}}{\min \left(N_{t}, N_{r}\right) !} .
\end{aligned}
$$

If $N_{t}<N_{r}$, we get

$$
\begin{aligned}
F_{\gamma_{\text {eq } 2}}(x) & \simeq-N_{r} \sum_{k=0}^{N_{r}-1} \frac{(-1)^{k}\left(\begin{array}{c}
N_{r}-1 \\
k
\end{array}\right)}{1+k} \\
& \times \frac{(-x)^{N_{t}}}{\left(N_{t}-1\right) !} \sum_{l=0}^{N_{t}-1}(-1)^{l}\left(\begin{array}{c}
N_{t}-1 \\
l
\end{array}\right)(1+l)^{N_{t}-1} \\
& \simeq x^{N_{t}}+O\left(x^{N_{t}}\right) .
\end{aligned}
$$

If $N_{r}<N_{t}$, similarly we get

$$
\begin{aligned}
F_{\gamma_{e q 2}}(x) & \simeq-N_{t} \sum_{k=0}^{N_{t}-1} \frac{(-1)^{k}\left(\begin{array}{c}
N_{t}-1 \\
k
\end{array}\right)}{1+k} \\
& \times \frac{(-x)^{N_{r}}}{\mu^{N_{r}}\left(N_{r}-1\right) !} \sum_{l=0}^{N_{r}-1}(-1)^{l}\left(\begin{array}{c}
N_{r}-1 \\
l
\end{array}\right)(1+k)^{N_{r}-1} \\
& \simeq \mu^{-N_{r}} x^{N_{r}}+O\left(x^{N_{r}}\right) .
\end{aligned}
$$

Finally, if $N_{t}=N_{r}=N$, we rewrite (41) as

$$
\begin{aligned}
F_{\gamma_{e q 2}}(x) & \simeq-N^{2}(-x)^{N} \sum_{l=0}^{N_{t}-1} \frac{(-1)^{l}\left(\begin{array}{c}
N-1 \\
l
\end{array}\right)}{1+l} \\
& \times \sum_{k=0}^{N-1} \frac{(-1)^{k}\left(\begin{array}{c}
N-1 \\
k
\end{array}\right)}{1+k} \frac{(1+l)^{N}+\left(\frac{1+k}{\mu}\right)^{N}}{N !} \\
& \simeq\left(1+\frac{1}{\mu^{N}}\right) x^{N}+O\left(x^{N}\right) .
\end{aligned}
$$

Substituting $x=\frac{\gamma_{T}}{\bar{\gamma}_{1}}$ into (42)-(44) gives the result in (10) and the proof is completed.

\section{REFERENCES}

[1] J. Boyer, D. D. Falconer, and H. Yanikomeroglu, "Multihop diversity in wireless relaying channels," IEEE Trans. Commun., vol. 52, pp. 18201830, Oct. 2004.

[2] M. O. Hasna and M.-S. Alouini, "A performance study of dual-hop transmissions with fixed gain relays," IEEE Trans. Wireless Commun., vol. 3, pp. 1963-1968, Nov. 2004.

[3] H. Mheidat and M. Uysal, "Impact of receive diversity on the performance of amplify-and-forward relaying under APS and IPS power constraints," IEEE Commun. Lett., vol. 10, pp. 468-470, June 2006.

[4] Y. Fan and J. Thompson, "MIMO configurations for relay channels: Theory and practice," IEEE Trans. Wireless Commun., vol. 6, pp. 17741786, May 2007.

[5] A. Adinoyi and H. Yanikomeroglu, "Cooperative relaying in multiantenna fixed relay networks," IEEE Trans. Wireless Commun., vol. 6, Feb. 2007, pp. 533-544.

[6] R. H. Y. Louie, Y. Li, and B. Vucetic, "Performance analysis of beamforming in two hop amplify and forward relay networks," in Proc. IEEE ICC 2008, Beijing, China, May 2008, pp. 4311-4315.

[7] D. B. da Costa and S. Aïssa, "Beamforming in dual-hop fixed gain relaying systems" in Proc. IEEE ICC 2009, Dresden, Germany, June 2009, pp. 1-5.

[8] S. W. Peters and R. W. Heath, Jr., "Nonregenerative MIMO relaying with optimal transmit antenna selection," IEEE Signal Proc. Lett., vol. 15, pp. 421-424, 2008.

[9] S. Chen, W. Wang, X. Zhang and D. Zhao, "Performance of amplifyand-forward MIMO relay channels with transmit antenna selection and maximal-ratio combining," in Proc. IEEE WCNC 2009, Budapest, Hungary, Apr. 2009, pp. 1-6.

[10] J.-B. Kim and D. Kim, "BER analysis of dual-hop amplify-and-forward MIMO relaying with best antenna selection in Rayleigh fading channels," IEICE Trans. Commun., vol. E91-B, pp. 2772-2775, Aug. 2008.

[11] N. R. Sollenberger, "Diversity and automatic link transfer for a TDMA wireless access link," in Proc. IEEE GLOBECOM 1993, Houston, TX, Dec. 1993, pp. 532536.

[12] I. S. Gradshteyn and I. M. Ryzhik, Table of Integrals, Series and Products. 6th ed., San Diego: CA, Academic Press, 2000.

[13] Z. Wang and G. B. Giannakis, "A simple and general parameterization quantifying performance in fading channels," IEEE Trans. Commun., vol. 51, pp. 1389-1398, Aug. 2003.

[14] A. P. Prudnikov, Y. A. Brychkov and O. I. Marichev, Integrals and Series. vol. 1, New York: NY, Gordon and Breach Science Publishes, 1986.

[15] M. Chiani, D. Dardari and M. K. Simon, "New exponential bounds and approximations probability in fading channels," IEEE Trans. Wireless Commun., vol. 2, pp. 840-845, July 2003. 